\title{
MOTIVICITY OF THE MIXED HODGE STRUCTURE OF SOME DEGENERATIONS OF CURVES
}

\author{
Hi-Joon Chae and Byungheup Jun
}

\begin{abstract}
We consider a degeneration of genus 2 curves, which is opposite to maximal degeneration in a sense. Such a degeneration of curves yields a variation of mixed Hodge structure with monodromy weight filtration. The mixed Hodge structure at each fibre, which is different from the limit mixed Hodge structure of Schmid and Steenbrink, can be realized as $H^{1}$ of a noncompact singular elliptic curve. We also prove that the pull back of the above variation of mixed Hodge structure to a double cover of the base space comes from a family of noncompact singular elliptic curves.
\end{abstract}

\section{Introduction}

A (pure) Hodge structure of weight $k$ consists of a free abelian group $V_{\mathbb{Z}}$ of finite rank together with a decreasing filtration $F^{\bullet}$ (called Hodge filtration) on $V_{\mathbb{C}}=V_{\mathbb{Z}} \otimes \mathbb{C}$ such that for each $p, V_{\mathbb{C}}=F^{p} \oplus \overline{F^{k-p+1}}$. A classical and most important example of Hodge structure of weight $k$ is the cohomology group $H^{k}(X, \mathbb{Z})$ (modulo torsion) of a compact Kähler manifold or a complete nonsingular variety $X$ defined over $\mathbb{C}$. The subspace $F^{p} \subset H^{k}(X, \mathbb{C})$ is generated by the classes representable by closed $(r, k-r)$ forms with $r \geq p$.

For a noncomplete and/or singular complex variety, Deligne defined a mixed Hodge structure on the cohomology [3, 4]. A mixed Hodge structure (or MHS, in abbreviation) on a free abelian group $V_{\mathbb{Z}}$ of finite rank consists of an increasing filtration $W_{\bullet}$ (called weight filtration) on $V_{\mathbb{Q}}=V_{\mathbb{Z}} \otimes \mathbb{Q}$ and a decreasing filtration $F^{\bullet}$ (again called Hodge filtration) on $V_{\mathbb{C}}$ such that the Hodge filtration induces pure Hodge structure of weight $k$ on each graded component $\mathrm{Gr}_{k}^{W}=W_{k} / W_{k-1}$.

A family of compact Kähler manifolds $X_{s}(s \in S$ ) (or smooth projective complex varieties) over a base space $S$ is a holomorphic map $f: X \rightarrow S$ between

Received December 19, 2008; Revised November 9, 2009.

2000 Mathematics Subject Classification. 32G20, 32S40.

Key words and phrases. variation of mixed Hodge structure.

The first named author was supported by the Korea Research Foundation Grant funded by the Korean Government (MOEHRD) (KRF-2005-042-C00005) and the second named author was partially supported by KOSEF R01-2003-000-11634-0.

(C)2010 The Korean Mathematical Society 
complex manifolds which is proper and smooth with $X_{s}=f^{-1}(s)$. Such a family of manifolds $X_{s}$ gives a family $R^{k} f_{*} \mathbb{Z}$ of Hodge structures $H^{k}\left(X_{s}, \mathbb{Z}\right)$ whose Hodge filtrations $F_{s}^{\bullet}$ vary holomorphically with respect to the parameter $s \in S$. This is a classical example of a variation of Hodge structure on a complex manifold. A variation of Hodge structure of weight $k$ on a complex manifold $S$ consists of a local system $V_{\mathbb{Z}}$ of free abelian groups of finite rank together with a decreasing filtration $\mathcal{F}^{\bullet}$ (called Hodge filtration) of the holomorphic vector bundle $\mathcal{V}=V_{\mathbb{Z}} \otimes \mathcal{O}_{S}$ by holomorphic subbundles such that (1) the Hodge filtration induces pure Hodge structure of weight $k$ on each stalk of $V_{\mathbb{Z}}$ and (2) covariant derivatives (with respect to the flat connection associated to the local system $V_{\mathbb{Z}}$ ) of sections of $\mathcal{F}^{p}$ belong to $\mathcal{F}^{p-1}$ (Griffiths' transversality).

A family of compact Kähler manifolds (or smooth projective varieties) as above may degenerate into a singular manifold. If the base space is onedimensional, such a family of manifolds is given locally by a holomorphic map between manifolds $f: X \rightarrow D$ ( $D$, a small open disk), which is proper and smooth over $D^{*}=D-\{0\}$. After suitable blow-ups and cyclic base changes, we may assume that the singular fiber $X_{0}=f^{-1}(0)$ is reduced and its singularities are normal crossings. We call such a family a (one-parameter semistable) degeneration. Thus a degeneration yields a variation of Hodge structure on the punctured disk $D^{*}$.

A local system of vector spaces (or of free abelian groups) on a manifold yields, actually is equivalent to, a representation of the fundamental group of the manifold (on the stalk of the local system at a base point). Applying this to the VHS on $D^{*}$ associated to a degeneration $f: X \rightarrow D$, we get the monodromy operator $T$, the action of the generator of the fundamental group of $D^{*}$ corresponding to a counterclockwise loop around the origin, which acts on $H^{k}\left(X_{t}, \mathbb{C}\right)$ where $t \in D^{*}$ is a base point. The monodromy operator $T$ is quasiunipotent (i.e., $T^{m}$ is unipotent for some $m$ ) and after suitable base change, can be assumed to be unipotent. The nilpotent operator $N=-\log T$ on $H^{k}\left(X_{t}, \mathbb{Q}\right)$ yields a canonical increasing filtration $W$ • which is called weight filtration (See Section 2 for more detail). Although the Hodge filtration $F^{\bullet}$ and this weight filtration $W_{\bullet}$ on $H^{k}\left(X_{t}, \mathbb{Q}\right)$ do not always yield a mixed Hodge structure, there exists a filtration $F_{\text {lim }}^{\bullet}$ which together with $W_{\bullet}$ induces a MHS (called the limit mixed Hodge structure) on $H^{k}\left(X_{t}, \mathbb{Q}\right)[11,13]$. Actually, these filtrations $W_{\bullet}$ and $F_{\text {lim }}^{\bullet}$ for varing $t$ form filtrations $\mathcal{W}_{\bullet}$ and $\mathcal{F}_{\text {lim }}^{\bullet}$ by sublocal systems of $R^{\bullet} f_{*} \mathbb{Z} \otimes \mathbb{Q}$ and by holomorphic subbundles of $R^{\bullet} f_{*} \mathbb{Z} \otimes \mathcal{O}$, respectively, yield a variation of mixed Hodge structure on a small deleted neighborhood of 0 . The limit mixed Hodge structure is used to prove the exactness of some sequences arising from a degeneration of varieties and has further applications.

There are mixed Hodge structures which are originated from geometry, but which are not the cohomolgy groups of some varieties a priori. It should be of interest to investigate if these mixed Hodge structures are isomorphic to mixed Hodge structures attached to some varieties, or are motivic more generally. One example of such mixed Hodge structures is the limit mixed Hodge structure 
explained in the last paragraph. This limit mixed Hodge structure is known to be motivic $[9,(2.3)]$. As indicated in the last paragraph, given a degeneration $f: X \rightarrow D$, the monodromy weight filtration $W_{\bullet}$ and the original Hodge filtration $F^{\bullet}$ do not always make a mixed Hodge structures on the cohomology groups of fibers [6, Sections 3, 4], [7, pp. 70-74]. In [6], Deligne showed that if the degeneration is maximal (i.e., if the resulting mixed Hodge structure $\left(W_{\bullet}, F_{\text {lim }}^{\bullet}\right)$ on the cohomology of nonsingular fiber is of Hodge-Tate type), this $\left(W_{\bullet}, F^{\bullet}\right)$ is always a mixed Hodge structure. In loc. cit., while it is described how these structures are motivic in some cases, a counterevidence is suggested to the problem in full generality relying on another conjecture. In this paper, we investigate if this $\left(W_{\bullet}, F^{\bullet}\right)$ arising from some degeneration of curves forms a MHS, and in that case if this new MHS can be realized as $H^{1}$ of some curve (We emphasize that we are not considering the limit $\left.\operatorname{MHS}\left(W_{\bullet}, F_{\lim }^{\bullet}\right)\right)$.

Before we state our main results, we should remark that in the rest of this paper we will use the term motivic in a narrower sense as follows: We say a mixed Hodge structure is motivic if it is isomorphic to the usual MHS of cohomology of some (singular quasi-projective) variety. In the same way, we say a variation of mixed Hodge structures over a base space $S$ is motivic if it is given by a flat family of such varieties over $S$. In this paper, unless otherwise stated, cohomology groups $H^{k}(V)$ of a complex variety $V$ are always equipped with usual (pure or mixed) Hodge structures. When we mean other MHS on $H^{k}(V)$ such as limit MHS or another new MHS above, we denote it by subscript (See below).

We consider the following degeneration of genus 2 curves (This degeneration is, in a sense, opposite to the above mentioned maximal degeneration). Let $f: C^{*} \rightarrow D^{*}$ be the family of smooth projective curves

$$
\begin{aligned}
& C_{s}=f^{-1}(s): y^{2}=\left(x^{2}-s\right)\left(x-a_{1}\right)\left(x-a_{2}\right)\left(x-a_{3}\right) \\
& \quad\left(s \in D^{*}, \text { a small punctured disk }\right)
\end{aligned}
$$

where $a_{1}, a_{2}, a_{3}$ are holomorphic functions on $D$ such that $\pm \sqrt{s}, a_{1}, a_{2}, a_{3}$ are distinct on $D^{*}$ and $a_{1}, a_{2}, a_{3}$ remain distinct at $s=0$. Then the original Hodge filtration $F^{\bullet}$ and the above mentioned weight filtration $W_{\bullet}$ on $H^{1}\left(C_{s}\right)$ define a mixed Hodge structure (Proposition 7). Thus $H^{1}\left(C_{s}\right)$ is equipped with three Hodge structures: usual pure HS, the limit MHS and the above new MHS. To avoid confusion, let us denote each of them by $H^{1}\left(C_{s}\right), H^{1}\left(C_{s}\right)_{\lim }$ and $H^{1}\left(C_{s}\right)_{M}$, respectively. One of our main results is that this new MHS $H^{1}\left(C_{s}\right)_{M}$ can be realized as $H^{1}$ of some singular noncompact curve (Theorem 13).

The family $\left(R^{1} f_{*} \mathbb{Z}, \mathcal{W}_{\bullet}, \mathcal{F}^{\bullet}\right)$ of mixed Hodge structure $H^{1}\left(C_{s}\right)_{M}$ is a variation of mixed Hodge structure on $D^{*}$ (See the paragraph before Theorem 14). Since it is isomorphic to $H^{1}$ of a singular curve fiberwise, it is natural to ask if there is a (flat) family of singular curves $g: X \rightarrow D^{*}$ such that $R^{1} g_{*} \mathbb{Z}$ equipped with the natural mixed Hodge structure realizes the whole VMHS $\left(R^{1} f_{*} \mathbb{Z}, \mathcal{W}_{\bullet}, \mathcal{F}^{\bullet}\right)$. The answer is partially positive. We prove that if we take 
the pull-back of the VMHS $\left(R^{1} f_{*} \mathbb{Z}, \mathcal{W}_{\bullet}, \mathcal{F}^{\bullet}\right)$ to a double cover of $D^{*}$, then there is a family of curves which realizes this VMHS (Theorem 14).

To prove these results, we develop an analogue of Legendre formula for the period determinant of Jacobi elliptic functions. Then we calculate a differential equation which is satisfied by the extension parameter of $\mathbb{Z}(-1)$ by $W_{1}$ that fixes $W_{2} / W_{0}$.

The solution of this differential equation is a $2: 1$ function on the elliptic curve to $\mathbb{P}^{1}$ which resembles the cross ratio of 4 points on the Riemann sphere in Hodge theoretic manner. Thus it is a multivalued function on the Riemann sphere.

As a motivation and a guide of our study of degeneration of genus 2 curves, we first consider a degeneration of elliptic curves in Section 2. This already has been analyzed in other places $[2,6,15]$.

Acknowledgment. We thank to Hélène Esnault for asking the related questions and motivations. We are indepted to Claude Sabbah, Masanori Asakura and Vincent Maillot for valuable comments and discussions.

\section{A degeneration of elliptic curves}

We consider the following degeneration of elliptic curves $f: E \rightarrow D$ with fibers given by

$$
E_{s}=f^{-1}(s): y^{2}=\left(x^{2}-s\right)(x-1) \quad(s \in D)
$$

where $D$ is a small disc centered at the origin. The Hodge filtration of $H^{1}\left(E_{s}, \mathbb{C}\right)$ is given by

$$
F^{0}=H^{1}\left(E_{s}, \mathbb{C}\right) \supset F^{1}=\left\{\text { holomorphic differential forms on } E_{s}\right\}=\mathbb{C} \cdot \frac{d x}{y} .
$$

Let $\gamma$ and $\delta$ denote the usual cycles spanning $H_{1}\left(E_{s}, \mathbb{Z}\right)$ with $\gamma$ vanishing on the singular fiber $E_{0}$. More precisely, if we view the elliptic curve $E_{s}$ as a branched double cover of $\mathbb{C} \cup\{\infty\}$ with two branch cuts along segments joining $\{ \pm \sqrt{s}\}$ and $\{1, \infty\}$, respectively. Then $\gamma$ is the cycle lying above a small circle around the branch cut joining $\{ \pm \sqrt{s}\}$ and $\delta$ is a cycle such that $\{\gamma, \delta\}$ is a symplectic basis of $H_{1}\left(E_{s}, \mathbb{Z}\right)$. Intuitively, the cycle $\gamma$ vanishes (or is homologous to zero) in the singular fiber $E_{0}$. In other words, its image under the inclusion $E_{s} \rightarrow E$ vanishes in $H_{1}$ of the total space $E$.

As explained in the introduction, we have the monodromy operator $T$ acting on $H^{1}$ of the smooth fiber $E_{s}\left(s \in D^{*}=D-\{0\}\right)$. The matrix of $T$ with respect to the dual basis $\left\{\gamma^{*}, \delta^{*}\right\}$ is

$$
T=\left(\begin{array}{ll}
1 & 0 \\
1 & 1
\end{array}\right)
$$

This follows from the formula for Picard-Lefschetz transformation [2, Chapter 1]. A nilpotent endomorphism $N$ on a vector space $V$ induces a canonical increasing filtration, called the weight filtration of $N[5,(1.6 .1)]$. In the simplest case when $N^{2}=0$, the weight filtration is $W_{-2}=(0) \subset W_{-1}=$ 
Image $(N) \subset W_{0}=\operatorname{Kernel}(N) \subset W_{1}=V$. In our case under consideration, $N=\log T=T-I$ defines the weight filtration (with the indices shifted by one from the canonical filtration) on $H^{1}\left(E_{s}, \mathbb{Q}\right)$ given by

$$
\mathbb{Q} \cdot \delta^{*}=W_{0}=W_{1} \subset W_{2}=H^{1}\left(E_{s}, \mathbb{Q}\right) .
$$

Proposition 1. $\left(H^{1}\left(E_{s}\right), W_{\bullet}, F^{\bullet}\right)$ is a mixed Hodge structure.

Proof. The proof is straightforward. Since $c_{1}=\int_{\gamma} d x / y \neq 0$, we have $[d x / y]=$ $c_{1} \gamma^{*}+c_{2} \delta^{*} \notin W_{0}$ and $W_{0} \cap F^{1}=(0)$. Let $V$ be a complex vector space equipped with integral (or real) structure. If $F^{k}=V \supset F^{k+1}=0$ is a one-step filtration on $V, V$ is a pure Hodge structure of weight $2 k$ (of type $(k, k)$ ) with $F^{\bullet}$ as its Hodge filtration. This shows that $G r_{0}^{W}$ and $G r_{2}^{W}$ with induced Hodge filtrations are pure Hodge structures of types $(0,0)$ and $(1,1)$, respectively.

Thus $H^{1}\left(E_{s}\right)$ is equipped with three Hodge structures: usual pure HS, the limit MHS and the above new MHS. Let us denote each of them by $H^{1}\left(E_{s}\right), H^{1}\left(E_{s}\right)_{\lim }$ and $H^{1}\left(E_{s}\right)_{M}$, respectively. We claim that this family of mixed Hodge structures $H^{1}\left(E_{s}\right)_{M}\left(s \in D^{*}\right)$ can be realized as those of a suitable family of singular non-complete curves. As a mixed Hodge structure, $H^{1}\left(E_{s}\right)_{M}$ is an extension of $\mathbb{Z}(-1)$ by $\mathbb{Z}(0)$. Such extensions are classified by $\mathbb{C}^{*}[6,(7.1-2)]$. The extension

$$
0 \rightarrow \mathbb{Z}(0) \stackrel{\alpha}{\rightarrow} H \stackrel{\beta}{\rightarrow} \mathbb{Z}(-1) \rightarrow 0
$$

corresponding to $q \in \mathbb{C}^{*}$ is described as follows:

$$
\begin{aligned}
H_{\mathbb{C}} & =\mathbb{C}^{2}, \quad \text { basis } \quad e_{0}, e_{1}, \\
W_{0} & =\mathbb{Q} \cdot e_{1}, \quad F^{1}=\mathbb{C} \cdot e_{0}, \\
H_{\mathbb{Z}} & =\mathbb{Z} \cdot e_{1}+(2 \pi i)^{-1} \mathbb{Z} \cdot\left(e_{0}+\log q e_{1}\right) \subset H_{\mathbb{C}}, \\
\alpha(1) & =e_{1}, \quad \beta\left((2 \pi i)^{-1} e_{0}\right)=(2 \pi i)^{-1} .
\end{aligned}
$$

In our case of $H^{1}\left(E_{s}\right)_{M}$, we have

$$
\frac{1}{2 \pi i} \log q=\int_{\delta} \frac{d x}{y} / \int_{\gamma} \frac{d x}{y} .
$$

Let $A=\left\{a_{1}, a_{2}\right\}, B=\left\{b_{1}, b_{2}\right\} \subset \mathbb{P}^{1}(\mathbb{C})$ with $A \cap B=\emptyset$. And let $X$ be the quotient space $\left(\mathbb{P}^{1} \backslash A\right) / B$, which is a singular quasi-projective curve. The normalization of $X$ is $X^{\prime}=\mathbb{P}^{1} \backslash A$, the smooth compactification of $X^{\prime}$ is $\bar{X}^{\prime}=\mathbb{P}^{1}$ and $\bar{X}=\mathbb{P}^{1} / B$ is a compactification of $X$. The $H_{1}(X, \mathbb{Z})$ is generated by the cycle $\delta$ represented by a path $\delta^{\prime}$ in $X^{\prime}=\mathbb{P}^{1} \backslash A$ joining $b_{1}$ and $b_{2}$, and by a small circle $\varepsilon$ around $a_{2}$. By $[4,(10.3 .11)]$, we have

$$
\begin{aligned}
& W_{0}\left(H^{1}(X, \mathbb{Z})\right)=\operatorname{Ker}\left(H^{1}(\bar{X}, \mathbb{Z}) \rightarrow H^{1}\left(\mathbb{P}^{1}, \mathbb{Z}\right)\right)=H^{1}(\bar{X}, \mathbb{Z})=\mathbb{Q} \cdot \delta^{*}, \\
& F^{1}\left(H^{1}(X, \mathbb{C})\right)=H^{0}\left(\bar{X}, \bar{r}_{*} \Omega_{\mathbb{P}^{1}}^{1}\left(\log \left\{a_{1}, a_{2}\right\}\right)\right)=H^{0}\left(\mathbb{P}^{1}, \Omega_{\mathbb{P}^{1}}^{1}\left(\log \left\{a_{1}, a_{2}\right\}\right)\right) .
\end{aligned}
$$


Clearly, $H^{0}\left(\mathbb{P}^{1}, \Omega_{\mathbb{P}^{1}}^{1}\left(\log \left\{a_{1}, a_{2}\right\}\right)\right)$ is spanned by $\frac{d z}{\left(z-a_{1}\right)\left(z-a_{2}\right)}$ (or $\frac{d z}{z-a_{i}}$ if either $a_{1}$ or $a_{2}$ is $\left.\infty\right)$. Since $\int_{\varepsilon} \frac{d z}{\left(z-a_{1}\right)\left(z-a_{2}\right)}=\frac{2 \pi i}{a_{2}-a_{1}} \neq 0$, it follows that $W_{0} \cap F^{1}=(0)$ and $H^{1}(X, \mathbb{C})$ is a mixed Hodge structure of type $\{(0,0),(1,1)\}$. The complex parameter $q \in \operatorname{Ext}_{\mathrm{MH}}(\mathbb{Z}(-1), \mathbb{Z}(0))$ corresponding to this mixed Hodge structure is

$$
\begin{aligned}
\frac{1}{2 \pi i} \log q & =\int_{\delta^{\prime}} \frac{d z}{\left(z-a_{1}\right)\left(z-a_{2}\right)} / \int_{\varepsilon} \frac{d z}{\left(z-a_{1}\right)\left(z-a_{2}\right)} \\
& =\frac{1}{2 \pi i} \log \left(\frac{\left(b_{2}-a_{1}\right)\left(b_{1}-a_{2}\right)}{\left(b_{2}-a_{2}\right)\left(b_{1}-a_{1}\right)}\right) .
\end{aligned}
$$

Hence, $q$ is the cross ratio of $a_{1}, a_{2}, b_{1}, b_{2}$. We have proven the following.

Proposition 2. Any nontrivial extension of $\mathbb{Z}(-1)$ by $\mathbb{Z}(0)$ can be realized as $H^{1}\left(\left(\mathbb{P}^{1} \backslash A\right) / B, \mathbb{Z}\right)$ for suitable $A, B \subset \mathbb{P}^{1}(\mathbb{C})$ with $|A|=|B|=2$ and $A \cap B=\emptyset$.

Of course, the choice of $A$ and $B$ is not unique. But given $q \in \operatorname{Ext}_{\mathrm{MH}}(\mathbb{Z}(-1)$, $\mathbb{Z}(0)) \cong \mathbb{C}^{*}$, it is most convenient to take $A=\{q, 1\}$ and $B=\{0, \infty\}$ since the cross ratio of $q, 1,0, \infty$ is $q$.

Now consider our family $E_{s}$ of elliptic curves and let

$$
\tau=\tau(s)=\left(\int_{\delta} d x / y\right) /\left(\int_{\gamma} d x / y\right)
$$

denote the right hand side of $(1)$ and put $q=\exp (2 \pi i \tau)$. Then the two mixed Hodge structures $H^{1}\left(E_{s}\right)_{M}$ and $H^{1}\left(\left(\mathbb{P}^{1} \backslash\{q, 1\}\right) /\{0, \infty\}\right)$ are isomorphic. We have proven the following.

Proposition 3. $H^{1}\left(E_{s}\right)_{M}$ is of geometric origin. In other words, it is isomorphic to the mixed Hodge structure $H^{1}$ of a singular quasi-projective curve.

Remark 4. The mixed Hodge structure $H^{1}\left(E_{s}\right)_{M}$ can be realized as $H^{1}$ (equipped with usual MHS) of the singular fibre $E_{0}: y^{2}=x^{2}(x-1)$ minus two points. The map $\rho: \mathbb{P}^{1} \rightarrow E_{0}$ given by $t \mapsto\left(\frac{-4 t}{(t-1)^{2}}, \frac{4 i t(t+1)}{(t-1)^{3}}\right)$ induces an isomorphism between $\mathbb{P}^{1} /\{0, \infty\}$ and $E_{0}$. Hence $H^{1}\left(\left(\mathbb{P}^{1} \backslash\{q, 1\}\right) /\{0, \infty\}\right)$ and $H^{1}\left(E_{0} \backslash\{\rho(q), \rho(1)\}\right)$ are isomorphic.

But it is not true in general that given a degeneration of curves $f: C \rightarrow D$, the corresponding family of mixed Hodge structures $H^{1}\left(C_{s}\right)_{M}$ can be obtained from a family of open subcurves of the singular fibre $C_{0}$. See the next section for an example.

Remark 5 . The $j$-invariant of the elliptic curve $E_{s}$ is given by

$$
j(\tau)=q^{-1}+744+196884 q+21493760 q^{2}+\cdots=\frac{64(3 s+1)^{3}}{s(s-1)^{2}} .
$$

This gives $q$ as a (transcendental) function of $s$ around $s=0$ and $q=0$. The correspondence $s \mapsto q$ is locally one-to-one. 
Remark 6. The mixed Hodge structure $\left(H^{1}\left(E_{s}\right), W_{\bullet}, F^{\bullet}\right)$ is different from the limit mixed Hodge structure $\left(H^{1}\left(E_{s}\right), W_{\bullet}, F_{\text {nil }}^{\bullet}\right)$ considered in [11]. The map $s \mapsto F^{\bullet}$, identifying each fibre $H^{1}\left(E_{s}\right)$ of the locally constant sheaf $R^{1} \pi_{*} \mathbb{C}$, induces a multivalued holomorphic map from the puctured disk $D^{*}$ into the Grassmannian variety of full flags of $\mathbb{C}^{2}$. The map $s \mapsto \exp \left(-\frac{\log s}{2 \pi i} N\right) F^{\bullet}(s)$ gives a single valued holomorphic map from $D^{*}$ into the Grassmanian and has a limit $F_{\text {lim }}^{\bullet}$ as $s \rightarrow 0$. Put $F_{\text {nil }}^{\bullet}(s)=\exp \left(\frac{\log s}{2 \pi i} N\right) F_{\lim }^{\bullet}$ then $\left(H^{1}\left(E_{s}\right), W_{\bullet}, F_{\text {nil }}^{\bullet}\right)$ is a variation of mixed Hodge structures near $s=0$.

In our case, this implies that $\tau(s)-\frac{\log s}{2 \pi i}$ has a limit, say, $\tau_{0}$ as $s \rightarrow 0$ and that the complex parameter corresponding to $\left(H^{1}\left(E_{s}\right), W_{\bullet}, F_{\text {nil }}^{\bullet}\right)$ is $q=\exp \left(2 \pi i \tau_{0}\right) s$, which is an algebraic function of $s$. Some calculation shows that $\tau_{0}=-\frac{2 \ln 2}{\pi i}$.

\section{A degeneration of genus 2 curves}

In this section we consider the following degeneration of genus 2 curves $f: C \rightarrow D$. This means, in particular, that fibers $C_{s}$ over $D^{*}=D-\{0\}$ are smooth projective curves of genus 2 and the singular fiber $C_{0}$ is reduced and its singularities are normal crossings (See the Introduction for the use of the terminology degeneration in this paper).

(2) $C_{s}=f^{-1}(s): y^{2}=\left(x^{2}-s\right)\left(x-a_{1}(s)\right)\left(x-a_{2}(s)\right)\left(x-a_{3}(s)\right) \quad(s \in D)$

where $a_{1}, a_{2}$ and $a_{3}$ are holomorphic functions on $D$ such that $\pm \sqrt{s}, a_{1}(s), a_{2}(s)$ and $a_{3}(s)$ are distinct for each $s \in D^{*}$ and $0, a_{1}(0), a_{2}(0)$ and $a_{3}(0)$ are distinct.

The Hodge filtration of $H^{1}\left(C_{s}, \mathbb{C}\right)$ is given by

$$
\begin{aligned}
F^{0}=H^{1}\left(C_{s}, \mathbb{C}\right) \supset F^{1} & =H^{0}\left(C_{s}, \Omega^{1}\right) \\
& =\left\{\text { holomorphic differential forms on } C_{s}\right\}
\end{aligned}
$$

Let $\gamma_{1}, \gamma_{2}, \delta_{1}, \delta_{2}$ be cycles of $C_{s}$ given as follow. If we view the hyperelliptic curve $C_{s}$ as a branched double cover of $\mathbb{C} \cup\{\infty\}$ with three branch cuts along segments joining $\{ \pm \sqrt{s}\}\left\{a_{1}, a_{2}\right\}$ and $\left\{a_{3}, \infty\right\}$, respectively. Then $\gamma_{1}$ and $\gamma_{2}$ are cycles lying above small circles around the branch cuts joining $\{ \pm \sqrt{s}\}$ and $\left\{a_{1}, a_{2}\right\}$, respectively. And $\delta_{1}$ and $\delta_{2}$ are cycles such that $\left\{\gamma_{1}, \delta_{1}, \gamma_{2}, \delta_{2}\right\}$ is a symplectic basis of $H_{1}\left(C_{s}, \mathbb{Z}\right.$ ). The cycle $\gamma_{1}$ vanishes (or is homologous to zero) in the singular fiber $C_{0}$ : its image under the inclusion $C_{s} \rightarrow C$ vanishes in $H_{1}$ of the total space $C$.

As explained in Introduction and Section 2, we have the monodromy operator $T$ acting on $H^{1}$ of the smooth fiber $C_{s}\left(s \in D^{*}=D-\{0\}\right)$. The matrix of $T$ with respect to the dual basis $\left\{\gamma_{1}^{*}, \delta_{1}^{*}, \gamma_{2}^{*}, \delta_{2}^{*}\right\}$ is the $4 \times 4$ block diagonal matrix with

$$
\left(\begin{array}{ll}
1 & 0 \\
1 & 1
\end{array}\right)
$$


along the diagonal. And the nilpotent operator $N=\log T=T-I$ induces the following weight filtration on $H^{1}\left(C_{s}, \mathbb{Q}\right)$ (See Section 2 and references there).

$$
\begin{aligned}
W_{0}=\operatorname{Image}(N)=\left\langle\delta_{1}^{*}\right\rangle & \subset W_{1}=\operatorname{Kernel}(N)=\left\langle\delta_{1}^{*}, \gamma_{2}^{*}, \delta_{2}^{*}\right\rangle \\
& \subset W_{2}=H^{1}\left(E_{s}, \mathbb{Q}\right) .
\end{aligned}
$$

We have the following:

Proposition 7. $\left(H^{1}\left(C_{s}\right), W_{\bullet}, F^{\bullet}\right)$ is a mixed Hodge structure.

Proof. The proof is again straightforward. Let $\omega_{1}, \omega_{2}$ be two linearly independent holomorphic forms on $C_{s}$ (hence form a basis of $F^{1}$ ) which are normalized as in the following period matrix:

$$
\left(\begin{array}{cccc}
\int_{\gamma_{1}} \omega_{1} & \int_{\gamma_{2}} \omega_{1} & \int_{\delta_{1}} \omega_{1} & \int_{\delta_{2}} \omega_{1} \\
\int_{\gamma_{1}} \omega_{2} & \int_{\gamma_{2}}^{2} \omega_{2} & \int_{\delta_{1}} \omega_{2} & \int_{\delta_{2}} \omega_{2}
\end{array}\right)=\left(\begin{array}{cccc}
1 & 0 & \Omega_{11} & \Omega_{12} \\
0 & 1 & \Omega_{21} & \Omega_{22}
\end{array}\right) .
$$

It is well known that $\Omega=\left(\Omega_{i j}\right)_{i, j=1,2}$ is symmetric and $\operatorname{Im} \Omega$ is positive definite. One can directly check that $\omega_{2} \in W_{1}$ since $\int_{\gamma_{1}} \omega_{2}=0$. Meanwhile $\omega_{1} \in W_{2} \backslash W_{1}$ from the nonvanishing of $\int_{\gamma_{1}} \omega_{1}$.

Hence $G r_{0}^{W}=W_{0}=\left\langle\delta_{1}^{*}\right\rangle=F^{0} \cap W_{0}=F^{0} \cap W_{0} \oplus \overline{F^{1} \cap W_{0}}$ is a pure Hodge structure of type $(0,0)$.

We have $F^{1} \cap W_{1}=\left\langle\omega_{2}\right\rangle \not \supset W_{0} . \overline{F^{1}} \cap W_{1}=\overline{F^{1} \cap W_{1}}$ is also an onedimensional subspace not containing $W_{0}$. Hence $W_{1} / W_{0}=F^{1}\left(W_{1} / W_{0}\right) \oplus$ $\overline{F^{1}}\left(W_{1} / W_{0}\right)$ if $\left(F^{1} \cap W_{1}+W_{0}\right) \cap\left(\overline{F^{1}} \cap W_{1}\right)=0$. This holds since the $\Omega=\left(\Omega_{i j}\right)$ above is a symmetric matrix whose imaginary part is positive definite, hence in particular $\Omega_{22} \notin \mathbb{R}$. So $G r_{1}^{W}$ with induced Hodge filtration is a pure Hodge structure of type $(1,0)$ and $(0,1)$. Finally, $G r_{2}^{W}=F^{1}\left(W_{2} / W_{1}\right)$, which tells us that this is a pure Hodge structure of type $(1,1)$.

We will denote by $H^{1}\left(C_{s}\right)_{M}$ the above new MHS on $H^{1}\left(C_{s}\right)$. We want to construct a curve $X$ whose $H^{1}$ is, as a mixed Hodge structure, isomorphic to $H^{1}\left(C_{s}\right)_{M}$. For simplicity of notation, we will denote the periods in (5) as follow:

$$
\tau=\Omega_{22}, \quad \alpha=\Omega_{12}=\Omega_{21}, \quad \beta=\Omega_{11} .
$$

We view $H^{1}\left(C_{s}\right)_{M}$ as an iterated extension of pure Hodge structures $G r_{0}^{W}=$ $W_{0}, G r_{1}^{W}=W_{1} / W_{0}$ and $G r_{2}^{W}=W_{2} / W_{1}$. The proof of the last proposition shows that $G r_{0}^{W} \cong \mathbb{Z}(0), G r_{2}^{W} \cong \mathbb{Z}(-1)$ and that $G r_{1}^{W}$ is isomorphic to $H^{1}$ of an elliptic curve whose normalized period is $\tau=\Omega_{22}=\int_{\delta_{2}} \omega_{2} / \int_{\gamma_{2}} \omega_{2}$. Note that since the imaginary part of the normalized period matrix $\left(\Omega_{i j}\right)$ is positive definite, the imaginary part of $\tau=\Omega_{22}$ is positive. Hence $\tau$ is indeed a normalized period of some elliptic curve. Let $E_{\tau}$ be an elliptic curve over $\mathbb{C}$ which is isomorphic to $\mathbb{C} /(\mathbb{Z}+\tau \mathbb{Z})$ as complex manifolds. Thus the MHS $H^{1}\left(C_{s}\right)_{M}$ is built up from $\mathbb{Z}(0), H^{1}\left(E_{\tau}\right)$ and $\mathbb{Z}(-1)$ :

$$
0 \rightarrow \mathbb{Z}(0) \rightarrow W_{1} \rightarrow H^{1}\left(E_{\tau}\right) \rightarrow 0,
$$




$$
\begin{aligned}
0 & \rightarrow W_{1} \rightarrow H^{1}\left(C_{s}\right)_{M} \rightarrow \mathbb{Z}(-1) \rightarrow 0, \\
0 & \rightarrow H^{1}\left(E_{\tau}\right) \rightarrow W_{2} / W_{0} \rightarrow \mathbb{Z}(-1) \rightarrow 0, \\
0 & \rightarrow \mathbb{Z}(0) \rightarrow H^{1}\left(C_{s}\right)_{M} \rightarrow W_{2} / W_{0} \rightarrow 0 .
\end{aligned}
$$

We recall the following description of extension of mixed Hodge structures in [1]. This is a generalization of description of $\operatorname{Ext}_{\mathrm{MH}}(\mathbb{Z}(-1), \mathbb{Z}(0))$ given in the previous section. For a mixed Hodge structure $H$ of highest weight $\leq 2 p-1$, the generalized intermediate Jacobian $J^{p} H$ is defined by

$$
J^{p} H=\frac{H_{\mathbb{C}}}{F^{p}+H_{\mathbb{Z}}} .
$$

Let $A$ and $B$ be two mixed Hodge structures. Then $\operatorname{Hom}_{\mathbb{Z}}\left(A_{\mathbb{Z}}, B_{\mathbb{Z}}\right)$ equipped with the following filtrations $\left(W_{\bullet}, F^{\bullet}\right)$ is a MHS: $W_{m} \operatorname{Hom}(A, B)=\{f \in$ $\operatorname{Hom}(A, B) \mid f\left(W_{k} A\right) \subset W_{k+m} B$ for each $\left.k\right\}$ and $F^{m} \operatorname{Hom}(A, B)$ is defined similarly. Suppose that $A$ and $B$ are separated in the sense that $W_{m} A=A$ and $W_{m} B=0$ for some $m$. Then there is a natural isomorphism of groups

$$
\operatorname{Ext}_{\mathrm{MH}}(B, A) \cong J^{0} \operatorname{Hom}(B, A)
$$

where Ext $_{\mathrm{MH}}$ denotes the group of extensions in the category of mixed Hodges structures. The above correspondence is given as follows. For an extension

$$
0 \rightarrow A \rightarrow H \stackrel{\pi}{\rightarrow} B \rightarrow 0,
$$

choose sections $s_{F}$ and $s_{\mathbb{Z}}$ of $\pi$ which preserve Hodge filtrations and integral structures, respectively. Then $\psi=s_{F}-s_{\mathbb{Z}}$, viewed as a map from $B_{\mathbb{C}}$ to $A_{\mathbb{C}}$, modulo $F^{0} \operatorname{Hom}(B, A)+\operatorname{Hom}(B, A)_{\mathbb{Z}}$ is the corresponding point of $J^{0} \operatorname{Hom}(B, A)$.

In cases of $\operatorname{Ext}_{\mathrm{MH}}\left(H^{1}\left(E_{\tau}\right), \mathbb{Z}(0)\right)$ and $\operatorname{Ext}_{\mathrm{MH}}\left(\mathbb{Z}(-1), H^{1}\left(E_{\tau}\right)\right)$, which will be used later, these mean the following. It can be shown that any class of homomorphism $\psi: H^{1}\left(E_{\tau}\right) \rightarrow \mathbb{Z}(0)$ in $J^{0} \operatorname{Hom}\left(H^{1}\left(E_{\tau}\right), \mathbb{Z}(0)\right)$ can be represented by a map such that $\psi\left(\gamma^{*}\right)=\alpha$ and $\psi\left(\delta^{*}\right)=0$ for some $\alpha \in \mathbb{C}$, where $\gamma$ and $\delta$ are cycles which are images under $\mathbb{C} \rightarrow \mathbb{C} /(\mathbb{Z}+\tau \mathbb{Z}) \cong E_{\tau}$ of line segments joining $\{0,1\}$ and $\{0, \tau\}$ respectively. Two such maps $\psi$ and $\psi^{\prime}$ corresponding to $\alpha$ and $\alpha^{\prime}$ respectively are in the same class if and only if $\alpha \equiv \alpha^{\prime} \bmod \mathbb{Z}+\tau \mathbb{Z}$. Hence the extensions of $H^{1}\left(E_{\tau}\right)$ by $\mathbb{Z}(0)$ are classified by $\mathbb{C} /(\mathbb{Z}+\tau \mathbb{Z})$. The extension $H$ corresponding to $\alpha \in \mathbb{C}$ is given explicitly as follows:

$$
\begin{aligned}
& H_{\mathbb{C}}=\mathbb{C}^{3} \supset H_{\mathbb{Z}}=\mathbb{Z}^{3}, \quad \text { basis } e_{0}, e_{1}, e_{2}, \\
& W_{0}=\mathbb{Q} \cdot e_{0}, \quad F^{1}=\mathbb{C} \cdot\left(e_{1}+\alpha e_{0}+\tau e_{2}\right) .
\end{aligned}
$$

Similarly, any class of homomorphism $\psi: \mathbb{Z}(-1) \rightarrow H^{1}\left(E_{\tau}\right)$ in $J^{0} \operatorname{Hom}(\mathbb{Z}(-1)$, $\left.H^{1}\left(E_{\tau}\right)\right)$ can be represented by a map such that $\psi\left((2 \pi i)^{-1}\right)=\alpha \cdot \delta^{*}$ some $\alpha \in \mathbb{C}$ and the class of $\psi$ in $J^{0} \operatorname{Hom}\left(\mathbb{Z}(-1), H^{1}\left(E_{\tau}\right)\right)$ depends only on the class of $\alpha$ in $\mathbb{C} /(\mathbb{Z}+\tau \mathbb{Z})$. Thus the extensions of $\mathbb{Z}(-1)$ by $H^{1}\left(E_{\tau}\right)$ are classified by 
$\mathbb{C} /(\mathbb{Z}+\tau \mathbb{Z})$. The extension $H$ corresponding to $\alpha \in \mathbb{C}$ is given explicitly as follows:

$$
\begin{aligned}
& H_{\mathbb{C}}=\mathbb{C}^{3} \supset H_{\mathbb{Z}}=\mathbb{Z}^{3}, \quad \text { basis } e_{1}, e_{2}, e_{3}, \\
& W_{1}=\left\langle e_{1}, e_{2}\right\rangle, \quad F^{1}=\left\langle e_{1}+\tau e_{2}, e_{3}+\alpha e_{2}\right\rangle
\end{aligned}
$$

Under these identifications, the extensions (7) and (9) above forming $H^{1}\left(C_{s}\right)_{M}$ correspond to periods $\Omega_{21}$ and $\Omega_{12}$ of (5) respectively, which are equal and denoted by $\alpha$ in this paper. We claim that these periods are non-zero. Otherwise, as a (pure) Hodge structure, $H^{1}\left(C_{s}\right)$ is the direct sum of two Hodge structures $\left\langle\gamma_{1}^{*}, \delta_{1}^{*}\right\rangle$ and $\left\langle\gamma_{2}^{*}, \delta_{2}^{*}\right\rangle$, each of which is isomorphic to $H^{1}$ of some elliptic curve. By Torelli theorem for curves, $C_{s}$ itself is isomorphic to a disjoint union of two elliptic curves, which is a contradiction.

\section{Geometric realization}

In this section, we ask if the mixed Hodge structure $H^{1}\left(C_{s}\right)_{M}$ arising from degeneration of curves as in the previous section is coming from geometry. We will consider a singular curve $(E \backslash A, B)$ whose $H^{1}$ gives the mixed Hodge structure of Proposition 7, where $E$ is an elliptic curve and $A=\left\{a_{1}, a_{2}\right\}, B=$ $\left\{b_{1}, b_{2}\right\} \subset E$ with $A \cap B=\emptyset$.

Let $U=E \backslash A$. In the following diagram, all the cohomology groups are with $\mathbb{Z}$-coefficient and are equipped with the (usual) mixed Hodge structures. The maps are morphisms between MHS's and the horizontal and the vertical sequences are exact.

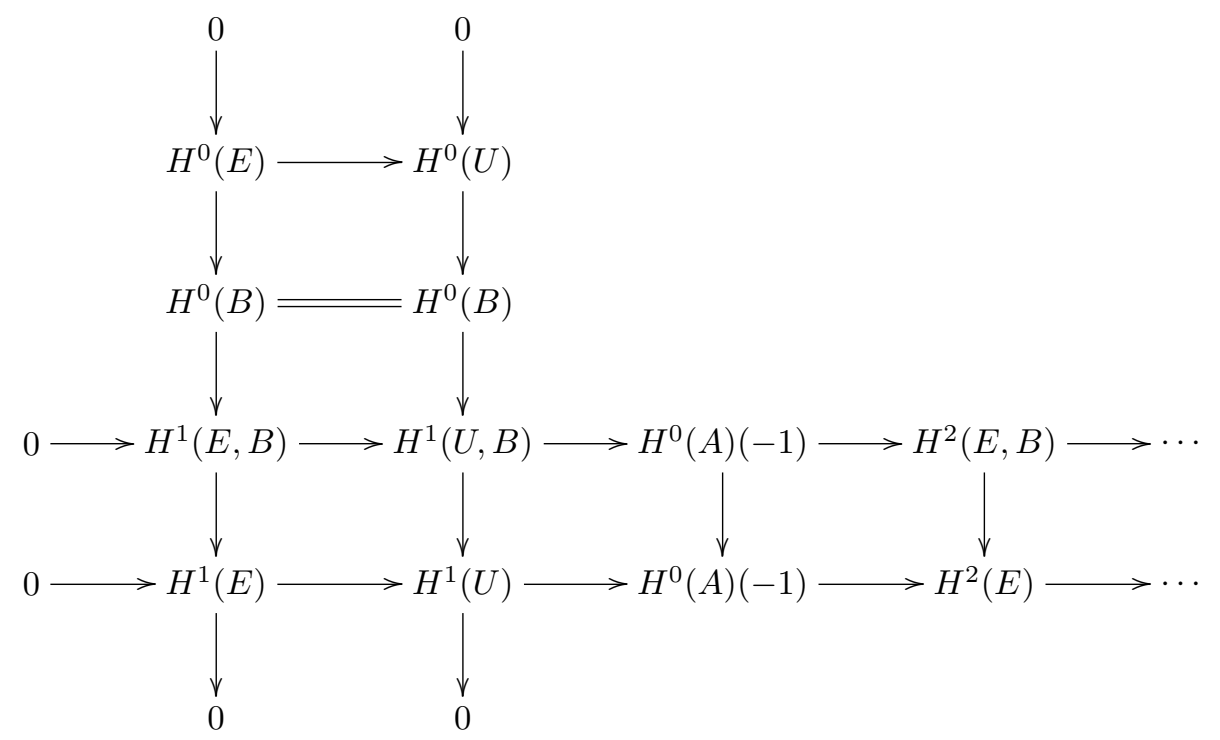


The vertical sequences are the long exact sequences coming from the normalization maps:

$$
\nu: E \rightarrow E / B \quad\left(\text { resp. }\left.\quad \nu\right|_{U}: U \rightarrow U / B\right)
$$

and the horizontal sequences are coming from the completion:

$$
j:(U, B) \rightarrow(E, B) .
$$

Note that any MHS $H$ of weight $\{0,1,2\}$ yields the following diagram of short exact sequences. Then compare these two diagrams (See also (13) and (14) below). For example, in the first column of the above diagram, $H^{0}(B)$ and $H^{1}(E)$ are pure of weight 0 and 1 , respectively. Hence the kernel of the map $H^{1}(E, B) \rightarrow H^{1}(E)$ is $W_{0}$ of $H^{1}(E, B)$ (actually of $H^{1}(U, B)$ which is shown below). Similarly, in the third row, $H^{1}(E, B)$ is mixed of weight $\{0,1\}$ and $H^{0}(A)(-1)$ is pure of weight 2 where ' $(-1)$ ' denotes the Tate twist. Hence $H^{1}(E, B)$, being the kernel of the map $H^{1}(U, B) \rightarrow H^{0}(A)(-1)$, is $W_{1}$ of $H^{1}(U, B)$.

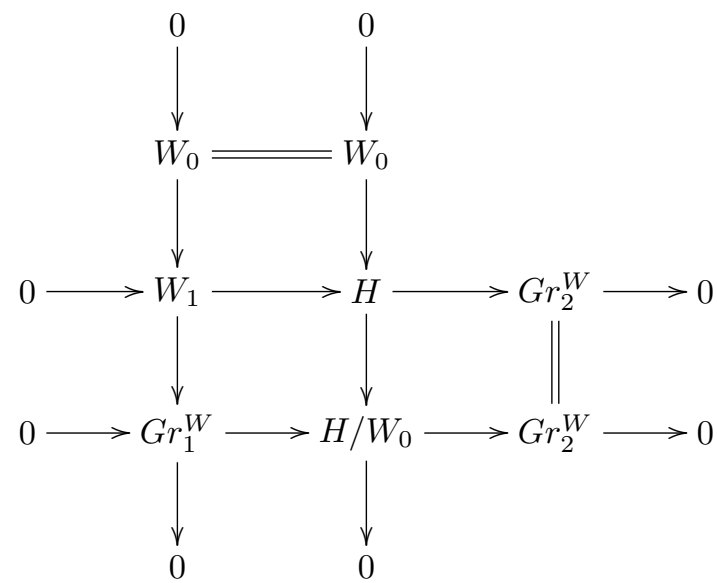

The four short exact sequences of MHS's appearing in the last diagram when $H$ is $H^{1}\left(C_{s}\right)_{M}$ of the last section, are nothing but the sequences $(7),(8),(9)$, and (10). Comparing last two diagrams, (7), (8), (9), and (10), and considering parameters describing extensions of MHS's in relevant short exact sequences, we seek for a singular curve $(E \backslash A, B)$ whose $H^{1}$ is isomorphic to $H^{1}\left(C_{s}\right)_{M}$. Recall that for $H^{1}\left(C_{s}\right)_{M}$, the normalized periods $\tau=\Omega_{22}, \alpha=\Omega_{12}=\Omega_{21}$ and $\beta=\Omega_{11}$ of $C_{s}$ in (5) are the parameters describing these extensions of MHS's.

As indicated in the last section, we have $G r_{1}^{W} H^{1}\left(C_{s}\right)_{M} \cong H^{1}\left(E_{\tau}\right)$ where $E_{\tau}$ is a complex elliptic curve, isomorphic to $\mathbb{C} /(\mathbb{Z}+\tau \mathbb{Z})$. So we take $E=E_{\tau}$. As usual, let $\gamma$ and $\delta$ be cycles of $E_{\tau}$ (or more precisely, of $\mathbb{C} /(\mathbb{Z}+\tau \mathbb{Z})$ ) which are images of line segments joining $\{0,1\}$ and $\{0, \tau\}$, respectively. And let $\omega$ be the invariant holomorphic differential form on $E_{\tau}$ corresponding to $d z$ on $\mathbb{C}$.

First, we will choose such $A, B \subset E_{\tau}$ separately in the following so that $H^{1}\left(E_{\tau}, B\right)$ realizes the extension of $H^{1}\left(E_{\tau}\right)$ by $\mathbb{Z}(0)$ in $(7)$ and $H^{1}\left(E_{\tau} \backslash A\right)$ 
realizes the extension of $\mathbb{Z}(-1)$ by $H^{1}\left(E_{\tau}\right)$ in $(9)$. The MHS of $W_{1}$ appears in the following diagram and (7).

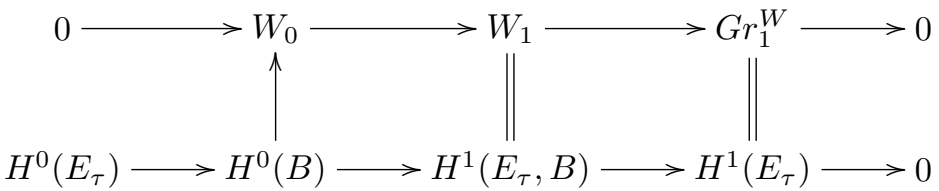

In view of (11) and remarks following it, we have to find $B=\left\{b_{1}, b_{2}\right\} \subset E_{\tau}$ such that

$$
\int_{\sigma_{b_{1}, b_{2}}} \omega=\alpha
$$

for a cycle $\sigma_{b_{1}, b_{2}}$ with $\partial \sigma_{b_{1}, b_{2}}=b_{2}-b_{1}$. Really, the cocycles $\delta_{b_{1}, b_{2}}^{*}$ and $\omega$ on $E_{\tau} / B$ are normalized generators of $W_{0} H^{1}\left(E_{\tau} / B\right)$ and $F^{1} H^{1}\left(E_{\tau} / B\right)$, respectively, appearing in (11). As $E_{\tau}$ is homogeneous and $\omega$ is invariant, we can put $b_{1}=0$. Let $\pi: \mathbb{C} \rightarrow E_{\tau}$ be the universal covering of $E_{\tau}$. Then $\pi^{*}(\omega)=d z$ if we set the parameter of $\mathbb{C}$ to $z$. Put $b_{2}=\pi(\alpha)$. Then we obtain, via change of variables,

$$
\int_{\sigma_{b_{1}, b_{2}}} \omega=\int_{0}^{\alpha} d z=\alpha
$$

as required. Actually, we have proven the following:

Proposition 8. In the category of mixed Hodge structures, any nontrivial extension of $H^{1}\left(E_{\tau}\right)$ by $\mathbb{Z}(0)$ can be realized as $H^{1}\left(E_{\tau} / B, \mathbb{Z}\right)$ for a suitable $B \subset E_{\tau}$ with $|B|=2$.

We turn to $W_{2} / W_{0}$, which comes from the following sequence and (9).

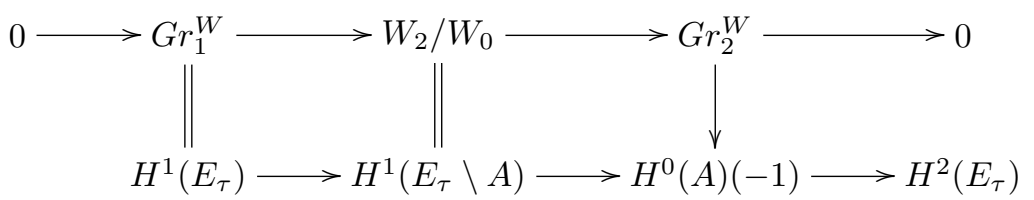

We have to find a configuration of $A=\left\{a_{1}, a_{2}\right\}$ in $E_{\tau}$ such that $H^{1}\left(E_{\tau} \backslash A\right)$ realizes the extension of $(9)$. Recall that the parameter describing this extension as given in (12) is $\alpha$. We can take cocycles $\gamma^{*}, \delta^{*}, \varepsilon^{*}$ on $E_{\tau} \backslash A$ as the normalized generators $e_{1}, e_{2}, e_{3}$ of $H^{1}\left(E_{\tau} \backslash A\right)$ given in (12) where $\varepsilon$ is a small circle around $a_{1}$. Recall that $F^{1} H^{1}\left(E_{\tau} \backslash A\right)$ is spanned by the holomorphic form $\omega$ and a meromorphic form $\omega_{A}$ on $E_{\tau}$ which is holomorphic on $E_{\tau} \backslash A$ and has simple poles at $A\left(\omega_{A}\right.$ is so called a differential of the third kind on $\left.E_{\tau}\right)$. Moreover if we normalize $\omega_{A}$ so that

$$
\operatorname{Res}_{a_{1}} \omega_{A}=\frac{1}{2 \pi i}
$$

then $\left\{\omega, \omega_{A}-\left(\int_{\gamma} \omega_{A}\right) \omega\right\}$ is the normalized basis of $F^{1}$ given in (12). And the parameter of the extension given in the second row of (14) is $\int_{\delta} \omega_{A}-$ 
$\left(\int_{\gamma} \omega_{A}\right)\left(\int_{\delta} \omega\right)$, which is the determinant of the period matrix

$$
\left(\begin{array}{cc}
\int_{\gamma} \omega & \int_{\delta} \omega \\
\int_{\gamma} \omega_{A} & \int_{\delta} \omega_{A}
\end{array}\right) .
$$

We calculate this period determinant applying the reciprocity law for differentials of the first and third kinds [8, p. 230]. Before doing so, we remark that the above period determinant is a pairing of homology and cohomology classes of $E_{\tau}$ if we view $\omega_{A}$ represents a cohomology class of $E_{\tau}$ as follows. Let $S$ be a path joining $a_{1}$ and $a_{2}$ which may be chosen so that it does not intersect $\gamma$ nor $\delta$. Since $\operatorname{Res}_{a_{1}} \omega_{A}+\operatorname{Res}_{a_{2}} \omega_{A}=0$, we may view $\omega_{A}$ as representing a class in $H^{1}\left(E_{\tau} \backslash S\right) \cong H^{1}\left(E_{\tau}\right)$, or more precisely, as its image under the map $H^{1}\left(E_{\tau} \backslash A\right) \rightarrow H^{1}\left(E_{\tau} \backslash S\right) \cong H^{1}\left(E_{\tau}\right)$. Then the above period determinant is equal to the pairing $\left\langle[\omega] \cup\left[\omega_{A}\right],\left[E_{\tau}\right]\right\rangle$.

Now suppose the elliptic curve $E_{\tau}$ is given explicitly in the following Weierstrass equation where $g_{2}$ and $g_{3}$ are Eisenstein series of weight 4 and 6 , respectively [12, Chapter 6].

$$
E_{\tau}: y^{2}=f_{\tau}(x):=4 x^{3}-g_{2}(\tau) x-g_{3}(\tau) .
$$

Then the map $\mathbb{C} /(\mathbb{Z}+\tau \mathbb{Z}) \rightarrow E_{\tau}$ given by $z \mapsto\left(\wp(z), \wp^{\prime}(z)\right)$ gives an isomorphism of complex Lie groups where $\wp(z)$ is the Weierstrass $\wp$-functions associated to the lattice generated by 1 and $\tau$. For the sake of simplicity of notation, we will not distinguish $\mathbb{C} /(\mathbb{Z}+\tau \mathbb{Z})$ and $E_{\tau}$ if no confusion arises. Because an elliptic curve is an abelian group, we may take $A=\left\{\left(x_{0}, \pm y_{0}\right)\right\}$. In other words, $a_{1}$ and $a_{2}$ are inverse to each other with respect to the addition. Then we have the following (Of course, $\omega_{A}$ is determined up to addition by a scalar multiple of $\omega$, which does not change the determinant of the above period matrix):

$$
\omega=\frac{d x}{y}, \quad \omega_{A}=\frac{y_{0}}{2 \pi i} \frac{d x}{\left(x-x_{0}\right) y} .
$$

Recall that $\pi: \mathbb{C} \rightarrow E_{\tau}, z \mapsto\left(\wp(z), \wp^{\prime}(z)\right)$ is the universal covering of $E_{\tau}$. We have

$$
\begin{aligned}
\pi^{*} \omega & =d z, \\
\pi^{*} \omega_{A} & =\frac{\sqrt{f_{\tau}(\wp(a))}}{2 \pi i(\wp(z)-\wp(a))} d z=\frac{\wp^{\prime}(a)}{2 \pi i(\wp(z)-\wp(a))} d z
\end{aligned}
$$

for $a$ in the fundamental domain of $E_{\tau}$ in $\mathbb{C}$ such that $\left(\wp(a), \wp^{\prime}(a)\right)=\left(x_{0}, y_{0}\right)$ (in other words, $\pi(a)=a_{1}$ and $\left.\pi(-a)=a_{2}\right)$. We obtain

$$
\begin{aligned}
\int_{\gamma} \omega \int_{\delta} \omega_{A}-\int_{\delta} \omega \int_{\gamma} \omega_{A} & =2 \pi i \sum_{c= \pm a} \operatorname{Res}_{c}\left(\int d z \cdot \pi^{*} \omega_{A}\right) \\
& =2 \pi i \sum_{c= \pm a} \operatorname{Res}_{c} \pi^{*} \omega_{A} \cdot \int_{0}^{c} d z
\end{aligned}
$$




$$
\begin{aligned}
& =2 \pi i \sum_{c= \pm a} \operatorname{Res}_{c} \frac{c \wp^{\prime}(a)}{2 \pi i(\wp(z)-\wp(a))} d z \\
& =2 a .
\end{aligned}
$$

Thus we obtain an analogue of Legendre formula for the period determinant of Jacobi elliptic functions.

Proposition 9. With notations as above, we have

$$
\operatorname{det}\left(\begin{array}{cc}
\int_{\gamma} \omega & \int_{\delta} \omega \\
\int_{\gamma} \frac{\wp^{\prime}(a)}{x-\wp(a)} \omega & \int_{\delta} \frac{\wp^{\prime}(a)}{x-\wp(a)} \omega
\end{array}\right)=4 \pi i a .
$$

Therefore putting $a=\alpha / 2$, i.e., putting $A=\{ \pm \alpha / 2\}$ in the second row of (14) we obtain the desired extension corresponding to $\alpha$. Note that $A$ is stable under involution of $E_{\tau}$, the choice of branch of the square root doesn't change the answer. We have proven the following:

Proposition 10. In the category of mixed Hodge structures, any nontrivial extension of $\mathbb{Z}(-1)$ by $H^{1}\left(E_{\tau}\right)$ can be realized as $H^{1}\left(E_{\tau} \backslash A, \mathbb{Z}\right)$ for a suitable $A \subset E_{\tau}$ with $|A|=2$.

Summing up, given non-zero $\alpha_{A}$ and $\alpha_{B}$ in $\mathbb{C} /(\mathbb{Z}+\tau \mathbb{Z})$ the mixed Hodge structures $H^{1}\left(E_{\tau} \backslash A\right)$ and $H^{1}\left(E_{\tau} / B\right)$ with $A=\left\{ \pm \alpha_{A} / 2\right\}$ and $B=\left\{z, z+\alpha_{B}\right\}$ (here the initial point $z$ can be arbitrary) correspond to $\alpha_{A} \in \operatorname{Ext}_{\mathrm{MH}}(\mathbb{Z}(-1)$, $\left.H^{1}\left(E_{\tau}\right)\right)$ and $\alpha_{B} \in \operatorname{Ext}_{\mathrm{MH}}\left(H^{1}\left(E_{\tau}\right), \mathbb{Z}(0)\right)$, respectively (Both of these extension groups can be identified with $\mathbb{C} /(\mathbb{Z}+\tau \mathbb{Z})$. See (11) and (12)). Now the final step is to analyze the MHS $H^{1}\left(E_{\tau} \backslash A, B\right)$. Recall that for $H^{1}\left(E_{\tau} \backslash A, B\right)$, we have

$$
\begin{aligned}
& W_{0} \cong \mathbb{Z}(0), \quad W_{1} \cong H^{1}(E, B), \quad G r_{1}^{W} \cong H^{1}\left(E_{\tau}\right), \\
& W_{2} / W_{0} \cong H^{1}\left(E_{\tau} \backslash A\right), \quad G r_{2}^{W} \cong \mathbb{Z}(-1) .
\end{aligned}
$$

Fixing $\alpha_{B}$ determines the MHS $H^{1}\left(E_{\tau} / B\right)$ and the short exact sequence $0 \rightarrow$ $\mathbb{Z}(0) \rightarrow H^{1}\left(E_{\tau}, B\right) \rightarrow H^{1}\left(E_{\tau}\right) \rightarrow 0$, which induces the long exact sequence

$$
\begin{aligned}
0 & \rightarrow \operatorname{Hom}_{M H}(\mathbb{Z}(-1), \mathbb{Z}(0)) \rightarrow \operatorname{Hom}_{M H}\left(\mathbb{Z}(-1), H^{1}\left(E_{\tau}, B\right)\right) \rightarrow \\
& \rightarrow \operatorname{Hom}_{M H}\left(\mathbb{Z}(-1), H^{1}\left(E_{\tau}\right)\right) \rightarrow \operatorname{Ext}_{\mathrm{MH}}(\mathbb{Z}(-1), \mathbb{Z}(0)) \rightarrow \\
& \rightarrow \operatorname{Ext}_{\mathrm{MH}}\left(\mathbb{Z}(-1), H^{1}\left(E_{\tau}, B\right)\right) \rightarrow \operatorname{Ext}_{\mathrm{MH}}\left(\mathbb{Z}(-1), H^{1}\left(E_{\tau}\right)\right) \rightarrow \cdots .
\end{aligned}
$$

Since $\operatorname{Hom}_{M H}\left(\mathbb{Z}(-1), H^{1}\left(E_{\tau}\right)\right)=0=\operatorname{Ext}_{\mathrm{MH}}^{2}(\mathbb{Z}(-1), \mathbb{Z}(0))$, we obtain the following short exact sequence which is the second row of the above long exact sequence.

$0 \rightarrow J^{0} \operatorname{Hom}(\mathbb{Z}(-1), \mathbb{Z}(0)) \rightarrow J^{0} \operatorname{Hom}\left(\mathbb{Z}(-1), H^{1}\left(E_{\tau}, B\right)\right) \rightarrow J^{0} \operatorname{Hom}\left(\mathbb{Z}(-1), H^{1}\left(E_{\tau}\right)\right) \rightarrow 0$.

We have $J^{0} \operatorname{Hom}(\mathbb{Z}(-1), \mathbb{Z}(0)) \cong \mathbb{C} / \mathbb{Z}(1) \cong \mathbb{C}^{*}$ and $J^{0} \operatorname{Hom}\left(\mathbb{Z}(-1), H^{1}\left(E_{\tau}\right)\right) \cong$ $E_{\tau}$. This sequence gives a $\mathbb{C}^{*}$-torsor over $E_{\tau}$. In our notations, $\alpha_{A}$ is a coordinate in the base space. We will show that given $\alpha_{A} \neq 0$, every extension which 
belongs to the fiber over $\alpha_{A}$ can be realized as $H^{1}\left(E_{\tau} \backslash A, B\right)$ for a suitable choice of the initial point $z$ in $B=\left\{z, z+\alpha_{B}\right\}$.

The fiber over $\alpha_{A}$ of the above $\mathbb{C}^{*}$-torsor and the action of $\mathbb{C}^{*}$ on the fiber are given as follow: let $\sigma_{z}$ be a cycle of $E_{\tau} \backslash A$ joining $z$ and $z+\alpha_{B}$. Any class of homomorphism $\psi: \mathbb{Z}(-1) \rightarrow H^{1}\left(E_{\tau}, B\right)$ in $J^{0} \operatorname{Hom}\left(\mathbb{Z}(-1), H^{1}\left(E_{\tau}, B\right)\right)$ which belongs to the fiber over $\alpha_{A}$ can be represented by a map such that $\psi\left((2 \pi i)^{-1}\right)=\beta \cdot \sigma_{z}^{*}+\alpha_{A} \cdot \delta^{*}$ for some $\beta \in \mathbb{C}$. The action of $\mathbb{C}^{*}$, or more precisely the action of $\mathbb{C}$ modulo $\mathbb{Z}$ is given by translation of the parameter $\beta$. The extension $H$ corresponding to $\beta$ is given explicitly as follows. Actually this gives any mixed Hodge structure $H$ with Hodge numbers $h^{0,0}=h^{0,1}=h^{1,0}=$ $h^{1,1}=1$.

$$
\begin{aligned}
& H_{\mathbb{C}}=\mathbb{C}^{3} \supset H_{\mathbb{Z}}=\mathbb{Z}^{4}, \quad \text { basis } e_{0}, e_{1}, e_{2}, e_{3}, \\
& W_{0}=\mathbb{Q} \cdot e_{0}, \quad W_{1}=\left\langle e_{0}, e_{1}, e_{2}\right\rangle, \\
& F^{1}=\left\langle\alpha_{B} e_{0}+e_{1}+\tau e_{2}, \beta e_{0}+\alpha_{A} e_{2}+e_{3}\right\rangle .
\end{aligned}
$$

For $H^{1}\left(E_{\tau} \backslash A, B\right)$ we can take $\sigma_{z}^{*}, \gamma^{*}, \delta^{*}, \epsilon^{*}$ (in previous notations) as $e_{0}, \ldots, e_{3}$, respectively. And $\omega, \omega_{A}-\left(\int_{\gamma} \omega_{A}\right) \omega$ as the normalized generators of $F^{1}$ above. Thus to show that any non-trivial extension of $\mathbb{Z}(-1)$ by $H^{1}\left(E_{\tau}, B\right)$ can be realized as $H^{1}\left(E_{\tau} \backslash A, B\right)$, it is enough to show that the following period, which is a multi-valued function of the initial point $z$, can take any complex value $(\operatorname{modulo} \mathbb{Z})$ :

$$
\beta_{z}:=\int_{\sigma_{z}} \omega_{A}=\int_{z}^{z+\alpha_{B}} \omega_{A} .
$$

This period depends on the homotopy class of $\sigma_{z}$ in $E_{\tau} \backslash A$, hence as a function of the initial point $z$, it is multi-valued. But $\exp \left(2 \pi i \beta_{z}\right)$ depends only on the initial point $z$ since the residues of $\omega_{A}$ stay in $\mathbb{Z}(-1)$. This (single-valued) function of $z$ is defined as long as $A \cap B=\emptyset$, that is, if $z \neq \pm \alpha_{A} / 2,-\alpha_{B} \pm \alpha_{A} / 2$. And clearly it is holomorphic in its domain of definition.

Proposition 11. With notations as before, the function $\exp \left(2 \pi i \beta_{z}\right)$ of $z$ is meromorphic on $E_{\tau}$. Its poles and zeros are as follow:

If $\alpha_{A} \neq \pm \alpha_{B}$, it has simple poles at $-\alpha_{B}-\alpha_{A} / 2, \alpha_{A} / 2$ and simple zeros at $-\alpha_{B}+\alpha_{A} / 2,-\alpha_{A} / 2$.

If $\alpha_{A}=\alpha_{B}$, simple poles at $-\alpha_{B}-\alpha_{A} / 2, \alpha_{A} / 2$ and a double zero at $-\alpha_{B}+$ $\alpha_{A} / 2=-\alpha_{A} / 2$.

If $\alpha_{A}=-\alpha_{B}$, a double pole at $-\alpha_{B}-\alpha_{A} / 2=\alpha_{A} / 2$ and simple zeros at $-\alpha_{B}+\alpha_{A} / 2,-\alpha_{A} / 2$.

Proof. The result is obtained by looking at the singularities of the differential equation satisfied by $2 \pi i \beta_{z}$. The function $2 \pi i \beta_{z}$ satisfies the following differential equation:

$$
\nabla_{d / d z}=\frac{d}{d z}-\frac{d}{d z} \log \left(\exp \left(2 \pi i \beta_{z}\right)\right)
$$




$$
=\frac{d}{d z}-\left(\frac{\wp^{\prime}\left(\alpha_{A} / 2\right)}{\wp\left(z+\alpha_{B}\right)-\wp\left(\alpha_{A} / 2\right)}-\frac{\wp^{\prime}\left(\alpha_{A} / 2\right)}{\wp(z)-\wp\left(\alpha_{A} / 2\right)}\right) .
$$

Recall that the Weierstrass $\wp$-functions associated to the lattice $\mathbb{Z}+\tau \mathbb{Z}$ is an even meromorphic function on $\mathbb{C} /(\mathbb{Z}+\tau \mathbb{Z})$ whose only singularity is a double pole at zero. From this analytic properties, it is easy to check the singularities of the above differential equation are simple poles at $\left\{ \pm \alpha_{A} / 2,-\alpha_{B} \pm \alpha_{A} / 2\right\}$.

If $\alpha_{A} \neq \pm \alpha_{B}$, then the residues at $-\alpha_{B}-\alpha_{A} / 2,-\alpha_{B}+\alpha_{A} / 2,-\alpha_{A} / 2, \alpha_{A} / 2$ are $-1,1,1,-1$, respectively. Really, we have

$$
\operatorname{Res}_{-\alpha_{B}-\alpha_{A} / 2} \nabla_{d / d z}=\frac{\wp^{\prime}\left(\alpha_{A} / 2\right)}{\wp^{\prime}\left(-\alpha_{A} / 2\right)}=-1
$$

and residues at other poles are obtained similarly. If $\alpha_{A}=\alpha_{B}$, then $-\alpha_{B}+$ $\alpha_{A} / 2=-\alpha_{A} / 2$ and the residue at this pole is 2 . Similarly, If $\alpha_{A}=-\alpha_{B}$, then $-\alpha_{B}-\alpha_{A} / 2=\alpha_{A} / 2$ and the residue at this pole is -2 . In all cases, the solution $\exp \left(2 \pi i \beta_{z}\right)$ of the above differential equation is a meromorphic function on $E_{\tau}$ with poles and zeros as described in the statement of the proposition.

Remark 12. We have seen in Section 2 that when we realize a non-trivial extension of $\mathbb{Z}(-1)$ by $\mathbb{Z}(0)$ as $H^{1}\left(\mathbb{P}^{1} \backslash\left\{a_{1}, a_{2}\right\},\left\{b_{1}, b_{2}\right\}\right)$, the cross ratio of the four points $a_{1}, a_{2}, b_{1}, b_{2}$ of $\mathbb{P}^{1}$ is the corresponding extension parameter. Similarly, given $\alpha_{A}, \alpha_{B}$ of $\mathbb{C} /(\mathbb{Z}+\tau \mathbb{Z})$, when we view $H^{1}\left(E_{\tau} \backslash\left\{ \pm \alpha_{A} / 2\right\},\{z, z+\right.$ $\left.\left.\alpha_{B}\right\}\right)$ as an iterated extension of $\mathbb{Z}(-1)$ by $H^{1}\left(E_{\tau}\right)$ and then by $\mathbb{Z}(0)$, the exponential period $\exp \left(2 \pi i \beta_{z}\right)$ is the corresponding extension parameter. Thus $\exp \left(2 \pi i \beta_{z}\right)$ may be thought to be an elliptic analogue of cross ratio of the four points $\alpha_{A} / 2,-\alpha_{A} / 2, z, z+\alpha_{B}$ of $E_{\tau}$.

Now we are able to show that the MHS $H^{1}\left(C_{s}\right)_{M}$ is isomorphic to $H^{1}\left(E_{\tau} \backslash\right.$ $A, B)$ for suitable $A, B \subset E_{\tau}$.

Theorem 13. Let $H=\left(H_{\mathbb{Z}}, W_{\bullet}, F^{\bullet}\right)$ be a mixed Hodge structure of weight 0 , 1, 2 such that $G r_{0}^{W}, G r_{1}^{W}$ and $G r_{2}^{W}$ are isomorphic to $\mathbb{Z}(0), H^{1}\left(E_{\tau}\right)$ and $\mathbb{Z}(-1)$, respectively, where $E_{\tau}$ is a complex elliptic curve. Suppose that in the category of mixed Hodge structures, the extension $W_{1}$ of $G r_{1}^{W}$ by $G r_{0}^{W}$ and the extension $W_{2} / W_{0}$ of $G r_{2}^{W}$ by $G r_{1}^{W}$ are non-trivial. Then $H$ is isomorphic to $H^{1}\left(E_{\tau} \backslash A, B\right)$ for suitable $A, B \subset E_{\tau}$ with $|A|=|B|=2$ and $A \cap B=\emptyset$. In particular, the mixed Hodge structure $H^{1}\left(C_{s}\right)_{M}$ in Proposition 7 can be realized in this way.

Proof. By the arguments given in paragraphs below Proposition 10 and before Proposition 11, it remains to show that the period $\beta_{z}$ in (18) can take any complex value (modulo $\mathbb{Z})$. By Proposition 11 , the exponential period map $E_{\tau} \rightarrow \mathbb{P}^{1}, z \mapsto \exp \left(2 \pi i \beta_{z}\right)$ is surjective and generically 2 to 1 . In particular, when restricted to $E_{\tau} \backslash\left\{ \pm \alpha_{A} / 2,-\alpha_{B} \pm \alpha_{A} / 2\right\}, \exp \left(2 \pi i \beta_{z}\right)$ is surjective onto $\mathbb{C}^{*}$. This proves the first statement of the theorem.

For the MHS $H^{1}\left(C_{s}\right)_{M}$ the parameters $\alpha_{A}$ and $\alpha_{B}$ in $\mathbb{C} /(\mathbb{Z}+\tau \mathbb{Z})$ corresponding to the extensions (9) and (7) are the same and equal to the period 
$\alpha=\Omega_{12}=\Omega_{21}$ of (5). As explained in the paragraph below (12), we have $\alpha \neq 0$. Hence the second statement of the theorem follows from the first.

The family of mixed Hodge structures $H^{1}\left(C_{s}\right)_{M}=\left(H^{1}\left(C_{s}\right), W_{\bullet}, F^{\bullet}\right)(s \in$ $\left.D^{*}\right)$ yields a variation of mixed Hodge structure $\mathcal{H}=\left(R^{1} f_{*} \mathbb{Z}, \mathcal{W}_{\bullet}, \mathcal{F}^{\bullet}\right)$ on $D^{*}$, where $f: C \rightarrow D^{*}$ is our family of curves $C_{s}=f^{-1}(s)$ (For a definition of variation of mixed Hodge structure, see $[14,(3.4)])$. Here $\mathcal{W}_{\bullet}$ are the sublocal systems of $R^{1} f_{*} \mathbb{Q}$ locally generated by the same bases of $W_{\bullet}$ given in Section 3 . Since the family $f$ is locally trivial, this makes sense. And $\mathcal{F}^{\bullet}$ is the usual Hodge filtration of $R^{1} f_{*} \mathbb{C} \otimes \mathcal{O}$. Since $\left(R^{1} f_{*} \mathbb{Z}, \mathcal{F}^{\bullet}\right)$ with Gauss-Manin connection is a variation of Hodge structure and each fiber $\left(H^{1}\left(C_{s}\right), W_{\bullet}, F^{\bullet}\right)$ is shown to be a MHS, it is obvious that $\left(R^{1} f_{*} \mathbb{Z}, \mathcal{W}_{\bullet}, \mathcal{F}^{\bullet}\right)$ is a VMHS. Now we ask the same question to the variation of the mixed Hodge structure that we have constructed: is there a (flat) family of singular curves over $D^{*}$ which realizes this VMHS?

Theorem 14. The pull back of the above variation of mixed Hodge structure $\mathcal{H}=\left(R^{1} f_{*} \mathbb{Z}, \mathcal{W}_{\bullet}, \mathcal{F}_{\bullet}\right)$ on $D^{*}$ to a double cover of $D^{*}$ can be realized by a family of singular affine elliptic curves $\left(E_{\tau(s)} \backslash A_{s}\right) / B_{s}$.

Proof. We will use the notations of Section 3. It boils down to the question whether the singular affine elliptic curve $\left(E_{\tau(s)} \backslash A_{s}\right) / B_{s}$ with $A_{s}=\{ \pm \alpha(s) / 2\}$ and $B_{s}=\{z(s), z(s)+\alpha(s)\}$, whose $H^{1}$ is shown to be isomorphic to $H^{1}\left(C_{s}\right)_{M}$ for each $s \in D^{*}$, can be glued to form a family over $D^{*}$. We can choose two global holomorphic differential forms $\omega_{1}, \omega_{2}$ on $C^{*}=C \backslash f^{-1}(0)$, whose restrictions to each fiber $C_{s}$ are linearly independent. Since $\gamma_{1}$ and $\gamma_{2}$ remain invariant under monodromy $T$, we may assume their restrictions to each fiber $C_{s}$ are normalized. Since $\delta_{2}$ is also invariant under monodromy, this shows that the parameters $\alpha(s)=\Omega_{21}(s)=\Omega_{12}(s)=\int_{\delta_{2}(s)} \omega_{1}(s)$ and $\tau(s)=\Omega_{22}(s)=$ $\int_{\delta_{2}(s)} \omega_{2}(s)$ are single-valued holomorphic functions on $D^{*}$. Thus $E_{\tau(s)} \backslash A_{s}$ form a flat family over $D^{*}$.

However, the initial point $z(s)$ of $B_{s}$, which measures the relative position of $A_{s}$ and $B_{s}$ is a multi-valued function of $s$. To see this, first recall that in view of (17) and the subsequent paragraph, $z=z(s)$ is such that $\Omega_{11}(s) \equiv \beta_{z}+$ const $\bmod \mathbb{Z}$, or equivalently such that $\exp \left(2 \pi i \Omega_{11}(s)\right)=\exp \left(2 \pi i \beta_{z}\right) \times$ const. Put $q(s)=\exp \left(2 \pi i \Omega_{11}(s)\right)$. This map sends $s=0$ to $q=0$ and locally one-to-one. If $z(s)$ is a single valued function of $s$, then around $q=0, z$ would be a single valued function of $q$. But from Proposition 11, we know this is impossible as there are exactly two $z$ 's mapping to one $q$. If we choose a double cover $D^{\prime}$ of $D^{*}, s^{\prime} \mapsto s$ such that $z\left(s^{\prime}\right)$ becomes a single-valued function of $s^{\prime}$ on $D^{\prime}$, then the pull back of $\mathcal{H}$ to this cover can be realized by the family of singular affine elliptic curves $\left.\left(E_{\tau\left(s^{\prime}\right)} \backslash A_{s^{\prime}}\right) / B_{s^{\prime}}\right)$. 


\section{References}

[1] J. Carlson, The geometry of the extension class of a mixed Hodge structure, Algebraic geometry, Bowdoin, 1985 (Brunswick, Maine, 1985), 199-222, Proc. Sympos. Pure Math., 46, Part 2, Amer. Math. Soc., Providence, RI, 1987.

[2] J. Carlson, S. Müller-Stach, and C. Peters, Period Mappings and Period Domains, Cambridge Studies in Advanced Mathematics, 85. Cambridge University Press, Cambridge, 2003.

[3] P. Deligne, Théorie de Hodge. II, Inst. Hautes Etudes Sci. Publ. Math. No. 40 (1971), $5-57$.

[4] , Théorie de Hodge. III, Inst. Hautes Etudes Sci. Publ. Math. No. 44 (1974), $5-77$.

[5] _ La conjecture de Weil. II, Inst. Hautes Etudes Sci. Publ. Math. No. 52 (1980), $137-252$.

[6] _ Local behavior of Hodge structures at infinity, Mirror symmetry, II, 683-699, AMS/IP Stud. Adv. Math., 1, Amer. Math. Soc., Providence, RI, 1997.

[7] P. Griffiths and L. Tu, Asymptotic behavior of a variation of Hodge structure, Topics in transcendental algebraic geometry (Princeton, N.J., 1981/1982), 63-74, Ann. of Math. Stud., 106, Princeton Univ. Press, Princeton, NJ, 1984.

[8] P. Griffiths and J. Harris, Princeples of Algebraic Geometry, Wiley, New York, 1978.

[9] L. Illusie, Autour du théorème de monodromie locale, Astérisque Vol. 223 (1994), 9-58.

[10] M. Kontsevich and D. Zagier, Periods, Mathematics unlimited-2001 and beyond, 771808, Springer, Berlin, 2001.

[11] W. Schmid, Variation of Hodge structure: the singularities of the period mapping, Invent. Math. 22 (1973), 211-319.

[12] J. Silverman, The Arithmetic of Elliptic Curves, Graduate Texts in Mathematics, 106. Springer-Verlag, New York, 1986.

[13] J. Steenbrink, Limits of Hodge structures, Invent. Math. 31 (1975/76), no. 3, 229-257.

[14] J. Steenbrink and S. Zucker, Variation of mixed Hodge structure. I, Invent. Math. 80 (1985), no. 3, 489-542.

[15] S. Müller-Stach, A remark on height pairings, Algebraic cycles and Hodge theory (Torino, 1993), 253-259, Lecture Notes in Math., 1594, Springer, Berlin, 1994.

Hi-JOON CHAE

Department of Mathematics Education

HONGIK UNIVERSITY

SeOul 121-791, KoreA

E-mail address: hchae@hongik.ac.kr

Byungheup Jun

Department of Mathematics

KONKUK UNIVERSITY

SEOUl 143-701, KoreA

E-mail address: byungheup@gmail.com 